\title{
Interleukin-6 upregulates SOXI8 expression in osteosarcoma
}

This article was published in the following Dove Press journal:

OncoTargets and Therapy

8 November 2017

Number of times this article has been viewed

\section{Zhong Wu* \\ Weizhi Yang* \\ Junjian Liu \\ Fan Zhang}

Department of Orthopedics, Shanghai Tenth People's Hospital, Tongji University School of Medicine, Shanghai, People's Republic of China

*These authors contributed equally to this work
Correspondence: Junjian Liu; Fan Zhang Department of Orthopedics, Shanghai Tenth People's Hospital, Tongji University School of Medicine, 30 I Yanchang Road, Shanghai 200072, People's Republic of China Tel +86 I85 02I 29363

Email liujunjian7679@।63.com; zhangf1I!@163.com
Aim: SOX18 is a potential oncogene in osteosarcoma via controlling osteosarcoma cell proliferation and metastasis. Interleukin-6 (IL-6), a major activator of Janus kinase 2 (JAK2)/ signal transducers and activators of transcription 3 (STAT3) signaling, plays an important role in the growth of carcinoma cells. The present study aims to investigate the correlation between IL-6 and SOX18 in osteosarcoma.

Materials and methods: Protein expression and mRNA expression were determined by Western blot and real-time polymerase chain reaction (PCR) analysis, respectively. Cell proliferation and apoptosis were identified by Cell Counting Kit- 8 assay and flow cytometry analysis, respectively.

Results: We found that SOX18, IL-6 and p-STAT3 were elevated in osteosarcoma compared with bone cyst tissues. A positive correlation between the mRNA levels of IL-6 and SOX18 was observed in osteosarcoma tissues. IL-6 stimulation dose dependently induced the mRNA and protein levels of SOX18 in U-2OS and MG63 cells. Furthermore, IL-6 significantly rescued the inhibitory and induction effects of SOX18 knockdown on osteosarcoma cell proliferation and apoptosis, respectively. The changes in cell proliferation (PCNA) and apoptosis-related proteins (Bcl-2, Bax and Cleaved-Caspase 3) were in line with the results of cell proliferation and apoptosis assays.

Conclusion: Our data suggest that IL-6 is a possible upstream regulator for SOX18 in osteosarcoma.

Keywords: IL-6, SOX18, osteosarcoma, proliferation, apoptosis

\section{Introduction}

Osteosarcoma is the most common malignant bone-forming tumor. ${ }^{1}$ Unlike other tumors, osteosarcoma usually occurs during the second and third decades of life. Despite the advances in surgery and chemotherapy for the past 2 decades, the survival time has hardly been improved. ${ }^{2}$ As such, understanding the molecular mechanisms involved in the development of osteosarcoma is crucial for developing new molecular targets of diagnosis and effective therapy.

SOX18 belongs to sex-determining region on the Y chromosome-related highmobility group box (SOX) gene family. ${ }^{3,4}$ SOX genes are highly conserved across species. SOX genes encode a group of transcription factors participating in developmental processes. ${ }^{5}$ Recently, several studies have revealed the role of SOX18 in carcinogenesis. SOX18 expression is elevated in gastric cancer ${ }^{6}$ and pancreatic ductal adenocarcinoma (PDAC). ${ }^{7}$ Overexpression of SOX18 indicates poor prognosis for a wide spectrum of human malignancies, such as gastric cancer, ${ }^{6}$ invasive ductal breast carcinoma, ${ }^{8}$ ovarian cancer ${ }^{9}$ and non-small cell lung cancer. ${ }^{10}$ In our previous paper, we have showed that SOX18 is upregulated in osteosarcoma and served as a potential 
oncogene in osteosarcoma via regulating osteosarcoma cell proliferation and metastasis. ${ }^{11}$ However, little is known about the upstream regulators of SOX18.

Interleukin-6 (IL-6), a proinflammatory cytokine, is a major activator of Janus kinase 2 (JAK2)/signal transducers and activators of transcription 3 (STAT3) signaling. ${ }^{12}$ STAT3 is a vital transcription factor that participates in the regulation of cell proliferation ${ }^{13}$ and cell apoptosis. ${ }^{14}$ It has been identified as an oncogene in a variety of tumor types. ${ }^{15}$ The total and phosphorylated forms of STAT3 are elevated in osteosarcoma cell lines and tissues. The increased level of phosphorylated STAT3 is associated with the poor prognosis in patients with osteosarcoma. ${ }^{16}$ Expression of other SOX genes, such as SOX2, ${ }^{17,18}$ is mediated by STAT3. However, whether IL-6/STAT3 regulates SOX18 expression in osteosarcoma is unknown.

In the present study, upregulation of IL-6 and SOX18 was observed in human osteosarcoma. The mRNA level of SOX18 had a positive correlation with that of IL-6 in osteosarcoma tissues. Cell proliferation and apoptosis assays suggested that the IL-6 was a possible upstream regulator for SOX18.

\section{Materials and methods}

\section{Tissue samples}

From 2011 to 2014, 50 patients with osteosarcoma and 20 patients with bone cyst admitted to Shanghai Tenth People's Hospital were enrolled in this study. This study was approved by the ethics committee of Shanghai Tenth People's Hospital, Tongji University. Written informed consent was obtained from every participant and complied with the guidelines of the ethics committee. Osteosarcoma and bone cyst samples were collected and immediately frozen in liquid nitrogen and stored at $-80^{\circ} \mathrm{C}$ until use.

\section{Real-time quantitative reverse transcription polymerase chain reaction (RT-PCR) assay}

The mRNA levels of SOX18 were evaluated by real-time quantitative RT-PCR assay as previously described. ${ }^{11}$ In brief, total RNA was extracted from tissue samples or cell lines with TRIzol Reagent (Thermo Fisher Scientific, Waltham, MA, USA) and then reverse transcribed into complementary DNA (cDNA) by using RevertAid First Strand cDNA Synthesis Kit (Thermo Fisher Scientific) per the manufacturer's instructions. Real-time PCR was carried out with SYBR Green PCR kit (Thermo Fisher Scientific) by ABI 7300 instrument (Thermo Fisher Scientific). SOX18 expression levels were determined by normalization to internal control, GAPDH.

\section{Western blot}

Tissue samples ( $200 \mathrm{mg}$ ) were ground into fine powder in liquid nitrogen using a mortar. Frozen tissue powder and cell lines were lysed in radio immunoprecipitation assay buffer (Beyotime, Shanghai, China) containing proteinase inhibitor cocktail (Sigma-Aldrich Co., St Louis, MO, USA), placed on ice for $30 \mathrm{~min}$ and then centrifuged at 13,000 rpm for $20 \mathrm{~min}$ at $4^{\circ} \mathrm{C}$. The collected supernatant was quantitated by BCA Protein Assay Kit (Thermo Fisher Scientific). Equal amounts of protein $(30 \mu \mathrm{g})$ was separated on sodium dodecyl sulfate polyacrylamide gel electrophoresis (SDS-PAGE) and transferred electrophoretically to a nitrocellulose membrane (EMD Millipore, Billerica, MA, USA). To block nonspecific binding, the membrane was incubated with $5 \%$ skim milk at room temperature for $30 \mathrm{~min}$. Following incubation with primary antibodies at $4^{\circ} \mathrm{C}$ overnight, the membrane was incubated with the corresponding horseradish peroxidase-conjugated secondary antibody (Beyotime). Protein expression was then analyzed using enhanced chemiluminescence (ECL; EMD Millipore) and ImageJ software (National Institutes of Health, Bethesda, MA, USA). GAPDH was detected as loading control. Sources of primary antibodies were as follows: 1) SOX18, p-STAT3, PCNA and Cleaved-Caspase 3 (Abcam, Cambridge, MA, USA); 2) STAT3 and GAPDH (Cell Signaling Technology, Danvers, MA, USA) and 3) Bcl-2 and Bax (Santa Cruz Biotechnology Inc., Dallas, TX, USA).

\section{Cell culture}

U-2OS, MG63 and HEK293T cells were obtained from American Type Culture Collection (Manassas, VA, USA). U-2OS cells were grown in Roswell Park Memorial Institute (RPMI) 1640 medium (Thermo Fisher Scientific), while MG63 and HEK293T cells were grown in the Dulbecco's Modified Eagle's Medium (DMEM; Thermo Fisher Scientific). Both media were supplemented with $10 \%$ fetal bovine serum (FBS; HyClone, Logan, UT, USA) and 1\% antibiotic (penicillin/streptomycin). All cell lines were maintained at $37^{\circ} \mathrm{C}$ in a $5 \% \mathrm{CO}_{2}$ atmosphere.

\section{Silencing of SOX 18 by short hairpin RNA (shRNA)}

shRNA targeting SOX18 (RNA interference [RNAi], TACC ACGTGGCACTGGCCATT) and a nonspecific scramble shRNA sequence (NC, TTCTCCGAACGTGTCACGTTT) was cloned into PLKO.1 (Addgene, Cambridge, MA, USA). 
Recombinant lentiviruses were produced by transfecting the lentiviral construct and package plasmids into HEK293T cells as described ${ }^{11}$ and collected to transduce U-2OS and MG63 cells.

\section{Cell Counting Kit-8 (CCK-8) assay}

Cell proliferation was evaluated by using CCK-8 Assay Kit (Beyotime) following the manufacturer's protocol. U-2OS and MG63 cells seeded in 96-well plates $(1,000$ 1,500 cells/well) were transduced with shRNAs and treated with or without $50 \mathrm{ng} / \mathrm{mL}$ IL-6 (Sigma-Aldrich Co.). After incubating for $0,24,48$ and $72 \mathrm{~h}, \mathrm{CCK}-8$ solution was added to each well and then incubated for $1 \mathrm{~h}$. Optical density (OD) values at the wavelength of $450 \mathrm{~nm}$ were measured with a microplate reader (Bio-Rad Laboratories Inc., Hercules, CA, USA). All experiments were conducted in triplicate and repeated at least three times.

\section{Cell apoptosis assay}

Cell apoptosis rate was assessed by Annexin V-fluorescein isothiocyanate (FITC) and propidium iodide (PI) staining kit (BD Biosciences, Franklin Lakes, NJ, USA). U-2OS and MG63 cells were transduced with shRNAs and treated with or without $50 \mathrm{ng} / \mathrm{mL}$ IL-6 (Sigma-Aldrich Co.) for $24 \mathrm{~h}$. At the end of culture period, both adherent and floating cells were harvested and then labeled with Annexin V-FITC and PI. Cell apoptosis was then analyzed using a FACScan instrument (BD Biosciences).

\section{Statistical analysis}

All data are presented as mean \pm SD. Statistical significance was determined by one-way analysis of variance
(ANOVA). A $P$-value $<0.05$ was considered as statistically significant.

\section{Results \\ SOXI8 expression was positively correlated with IL-6 expression in osteosarcoma tissues}

The mRNA expression of SOX18 and IL-6 was determined in osteosarcoma $(n=50)$ and control bone cyst tissues $(\mathrm{n}=20)$. Comparing with bone cyst tissues, SOX18 and IL-6 mRNA levels increased by $127.5 \%$ and $71.4 \%$, respectively (Figure 1A). Pearson correlation analysis was then performed to assess whether any relationship existed between the mRNA levels of SOX18 and IL-6 in osteosarcoma tissues. As shown in Figure 1B, the SOX18 mRNA level was positively correlated with the IL-6 level $(r=0.7446, P<0.001)$. The protein levels of SOX18, IL-6 and p-STAT3 were also evaluated in osteosarcoma and bone cyst tissues. Osteosarcoma tissues had higher protein levels of SOX18, IL-6 and p-STAT3 as compared to bone cyst tissues (Figure 1C). These data indicated an association between IL-6/STAT3 and SOX18 during osteosarcoma progression.

\section{IL-6/STAT3 induced SOX 18 expression}

To determine whether IL-6 affected SOX18 expression in osteosarcoma, we treated U-2OS and MG63 cells with recombinant IL- 6 and found that IL- 6 concentration dependently enhanced the mRNA and protein levels of SOX18 (Figure 2A). These data suggested that IL-6 may induce SOX18 expression at the transcriptional level. Moreover, IL-6 treatment increased the levels of p-STAT3 in a concentration-dependent manner (Figure 2B). Additional
A

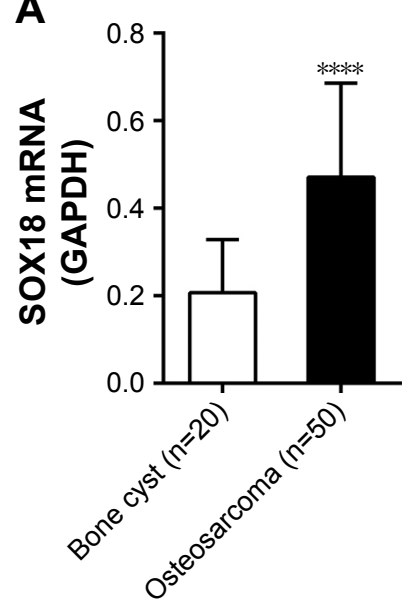

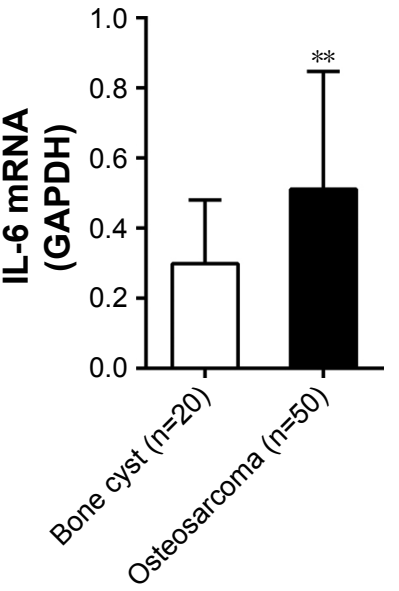

B

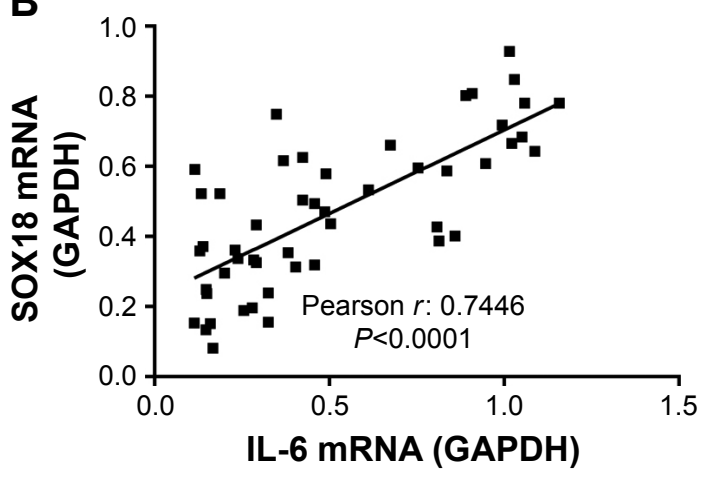

Figure I (Continued) 

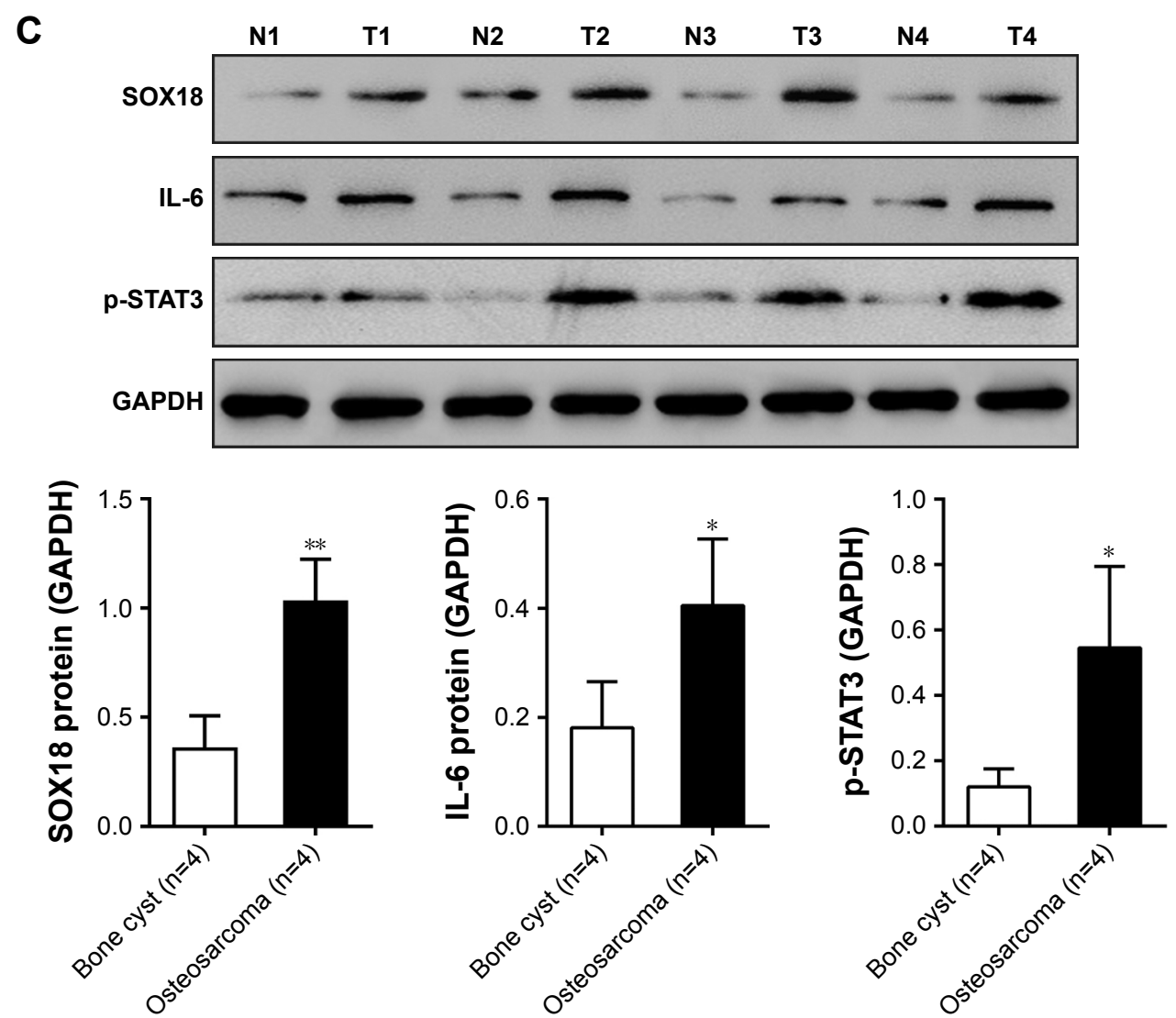

Figure I SOXI8 mRNA expression was correlated with IL-6 mRNA expression in osteosarcoma tissues.

Notes: (A) mRNA levels of SOXI8 and IL-6 were significantly higher in osteosarcoma tissues $(n=50)$ than in bone cyst tissues ( $\mathrm{n}=20)$. (B) Pearson correlation scatter plot of IL-6 and SOXI8. (C) Western blot analysis of SOXI8, IL-6 and P-STAT3. Representative blots and protein levels relative to GAPDH are shown. $* P<0.05$, $* * P<0.0$ I and $* * * * P<0.000$ I as compared with bone cyst tissues.

Abbreviation: IL-6, interleukin-6.

AG490 (a STAT3 inhibitor) exposure significantly attenuated IL-6-induced SOX18 expression (Figure 2C). These results suggested that IL-6/STAT3 induced SOX18 transcription in osteosarcoma cells.

\section{IL-6 promoted osteosarcoma cell proliferation via SOX 18}

Several investigators have reported that IL- 6 can modulate the proliferation of carcinoma cells. ${ }^{19-21}$ To investigate whether IL-6 exerted functions through SOX18, we knocked down SOX18 expression in two osteosarcoma cell lines, U-2OS and MG63, by RNAi as previously reported (Figure 3A). ${ }^{11}$ As displayed in Figure 3B and C, IL-6 exposure remarkably induced cell proliferation and such effect was notably attenuated by SOX18 knockdown.

\section{IL-6 exposure attenuated the induction effects of SOXI8 knockdown on osteosarcoma cell apoptosis}

Cell apoptosis was evaluated by Annexin V-FITC/PI staining assay. As shown in Figure 4A and B, knockdown of SOX18 in U-2OS or MG63 cells significantly induced cell apoptosis in comparison with corresponding scramble shRNA. The presence of IL-6 significantly reduced SOX18 knockdowninduced cell apoptosis.

\section{Expression of PCNA, Bcl-2, Bax and Cleaved-Caspase 3}

We detected the protein levels of a cell proliferation marker $\left(\mathrm{PCNA}^{22}\right)$ and cell apoptosis-associated proteins (Bcl-2, Bax and Cleaved-Caspase $3^{23,24}$ ). Consistent with the results of CCK-8 and Annexin V-FITC/PI staining assays, IL-6 stimulation enhanced the protein expression of PCNA and Bcl-2, which was reduced by SOX18 knockdown (Figure 5). The reversed effects were observed in Bax and CleavedCaspase 3 expression.

\section{Discussion}

Our recent study has reported that SOX18 is overexpressed in osteosarcoma and served as a potential oncogene in osteosarcoma via regulating osteosarcoma proliferation and metastasis. ${ }^{11}$ Several studies have concerned the upstream 


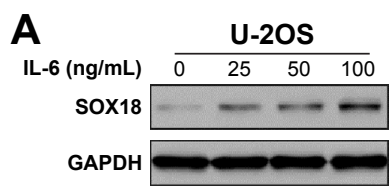

U-2OS

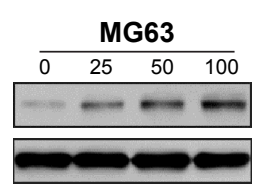

MG63

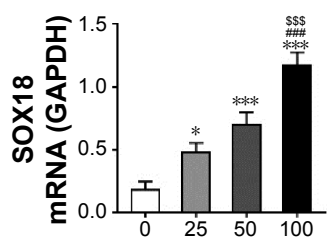

B

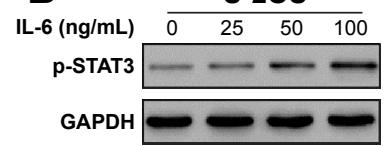

U-2OS

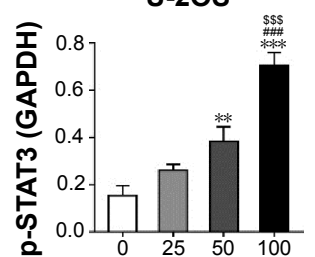

MG63

$\mathrm{IL}-6+\mathrm{IL}-6+$ WT DMSO AG490

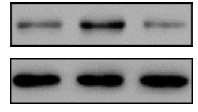

MG63

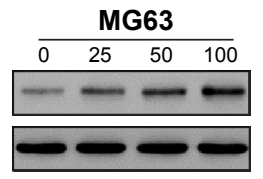

MG63

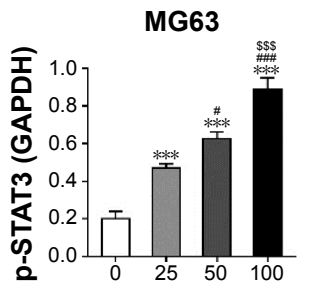

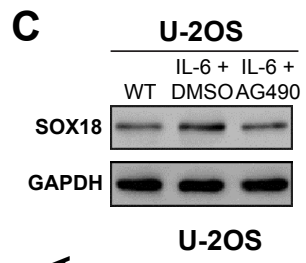
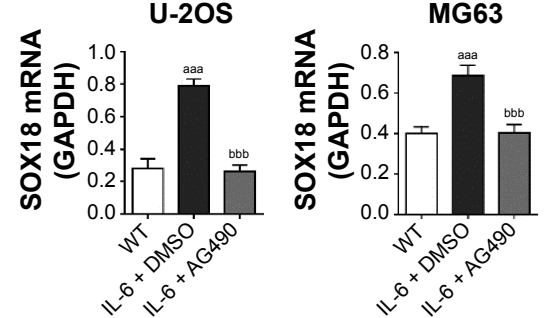

Figure 2 IL-6 increased SOX 18 expression.

Notes: (A and B) U-2OS and MG63 cells were exposed with increasing dose of IL-6 (0, 25, 50 and I00 ng/mL) for 24 h. Expression of SOXI8 protein (upper panel) and GAPDH (lower panel) was analyzed by Western blot and real-time PCR, respectively $(\mathbf{A})$. Western blot analysis of $\mathrm{P}-\mathrm{STAT3}$ is shown $(\mathbf{B})$. $* P<0.05$, $* * P<0.0 \mathrm{I}$ and

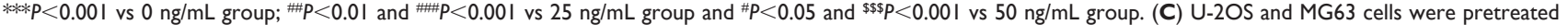
with $30 \mu$ M AG490 (Selleck Chemicals, Houston, TX, USA) or DMSO for I h and then exposed to $50 \mathrm{ng} / \mathrm{mL}$ IL-6 for $24 \mathrm{~h}$. Expression of SOXI8 protein (upper panel) and GAPDH (lower panel) was analyzed. aaa $P<0.001$ vs WT and bbt $P<0.001$ vs IL-6 + DMSO.

Abbreviations: PCR, polymerase chain reaction; DMSO, dimethyl sulfoxide; WT, without any treatment; IL-6, interleukin-6.

A

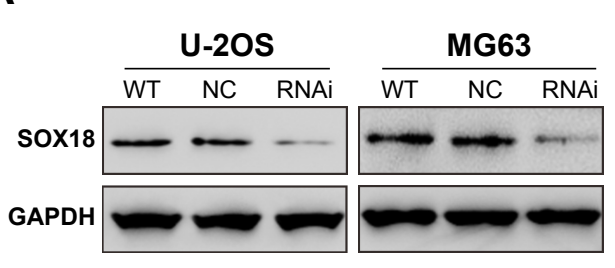

\section{B}

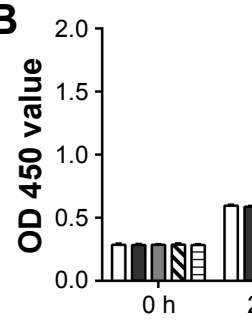

U-20S

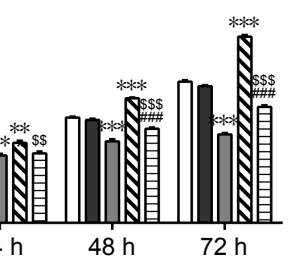

U-20S

MG63
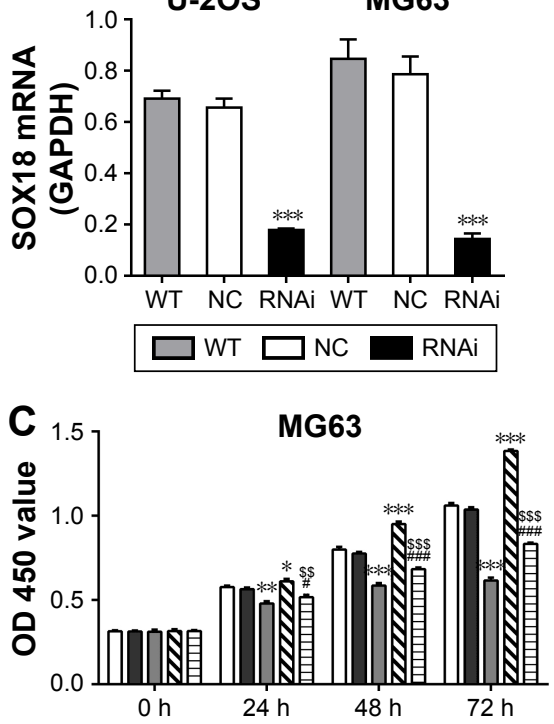

Figure 3 IL-6 promoted osteosarcoma cell growth via SOXI8.

Notes: (A) U-2OS and MG63 cells were transduced with SOXI8 shRNA (RNAi) or control shRNA (NC). At $48 \mathrm{~h}$ after transduction, expression of SOXI 8 protein (upper panel) and GAPDH (lower panel) was analyzed. (B) U-2OS and (C) MG63 cells seeded in 96-well plates were transduced with shRNAs and treated with or without $50 \mathrm{ng} / \mathrm{mL}$ IL-6. After incubating for $0,24,48$ and $72 \mathrm{~h}, \mathrm{CCK}-8$ assay was performed to determine cell proliferation. $* P<0.05$, $* * P<0.0$ I and $* * * P<0.00 \mathrm{I}$ vs $W T$; ${ }^{*} P<0.05$ and ${ }^{* \# P<0.00 I}$ vs RNAi and $\$ \$ P<0.01$ and $\$ \$ P<0.001$ vs $N C+I L-6$.

Abbreviations: RNAi, RNA interference; CCK-8, Cell Counting Kit-8; WT, without any treatment; OD, optical density; IL-6, interleukin-6. 


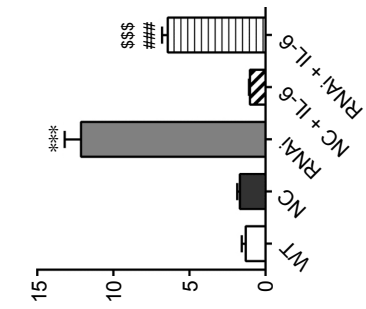

(\%) ә†е s!̣soldode К스
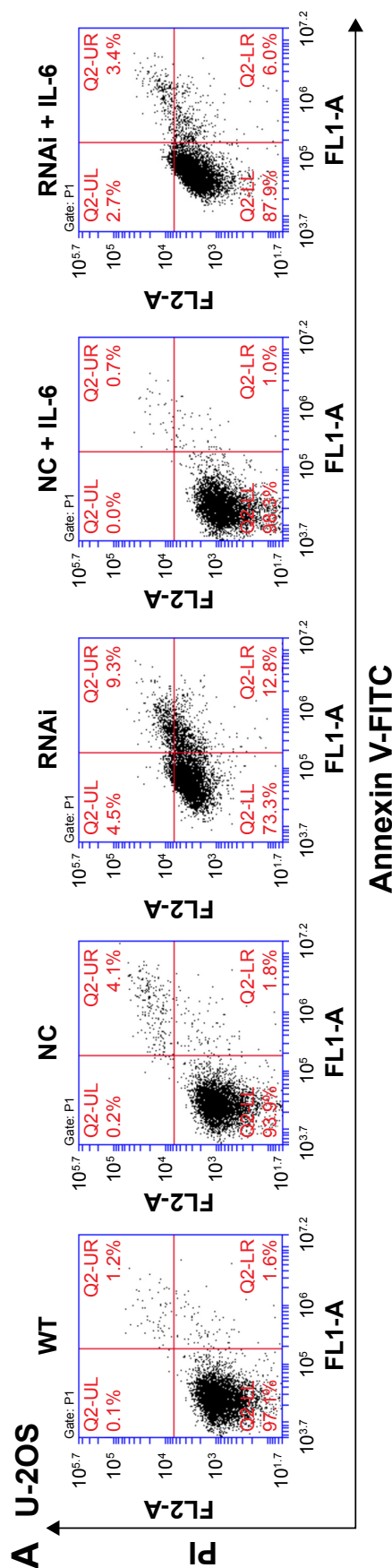

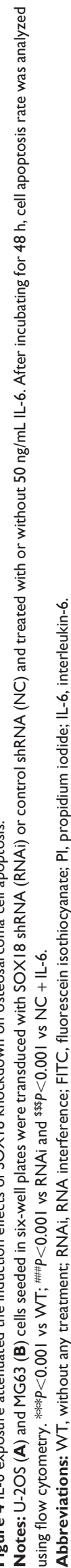


A

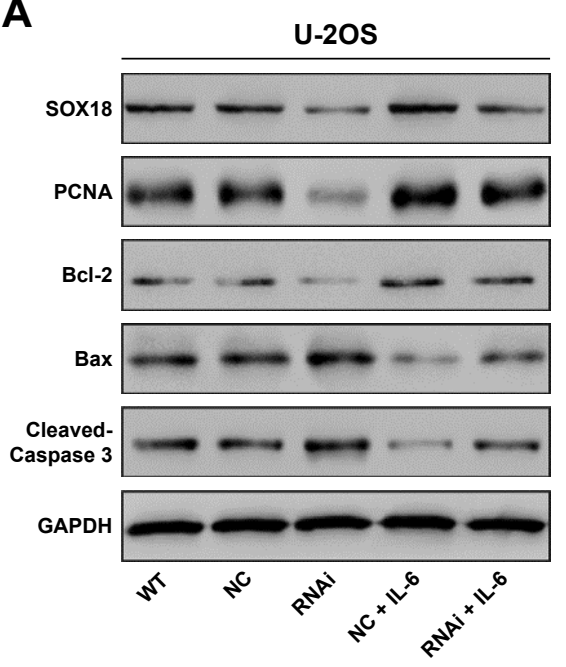

$\mathbf{B}$

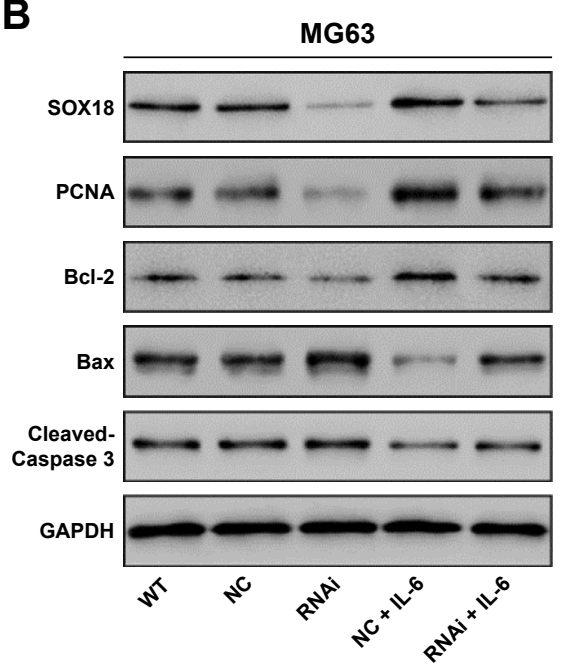

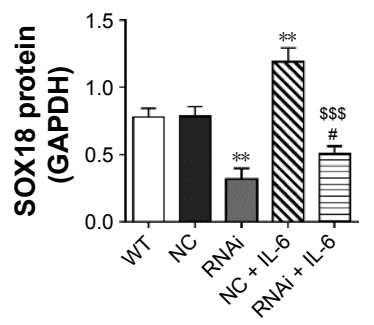
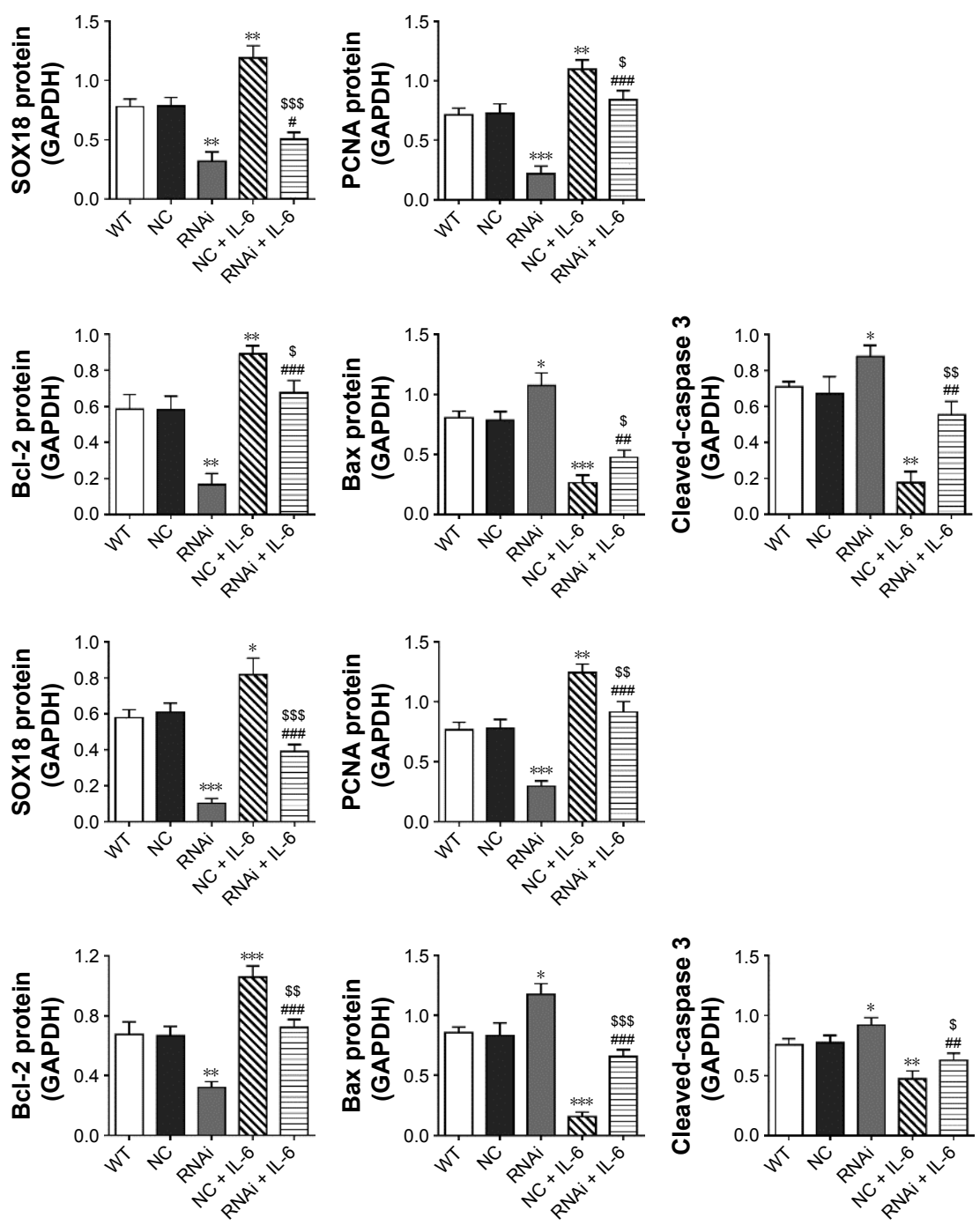

Figure 5 Expression of PCNA, Bcl-2, Bax and Cleaved-Caspase 3.

Notes: U-2OS (A) and MG63 (B) cells seeded in six-well plates were transduced with SOXI8 shRNA (RNAi) or control shRNA(NC) and treated with or without 50 ng/mL

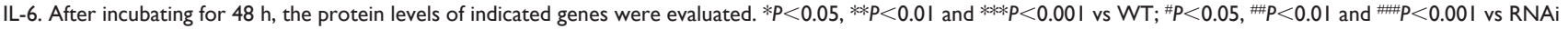
and $\$ P<0.05, \$ \$ P<0.01$ and $\$ \$ P<0.001$ vs $N C+$ IL-6.

Abbreviations: WT, without any treatment; RNAi, RNA interference; IL-6, interleukin-6; PCNA, cell proliferation.

regulators for SOX genes. For instance, SOX4 was induced by TGF- $\beta$ in Th 2 cells. ${ }^{25}$ SOX 2 expression was induced by STAT3 in the neural precursor cell and breast cancer cells. ${ }^{17,18}$ IL-6 expression was significantly higher in human osteosarcoma tissues than in the normal bone. ${ }^{26}$ As a major activator of JAK2/STAT3 signaling, ${ }^{12}$ IL-6 has been reported to modulate the proliferation of carcinoma cells. ${ }^{19-21}$ Thus, we tried to investigate the effects of IL-6 treatment on SOX18 expression. In the present study, elevated IL-6 and SOX18 expression were observed in osteosarcoma tissues as compared with bone cyst tissues at both mRNA and protein levels. The increased level of phosphorylated STAT3 is associated with poor prognosis of osteosarcoma. ${ }^{16}$ Presently, phosphorylated STAT3 was elevated in osteosarcoma tissues.
Moreover, SOX18 mRNA expression was strongly correlated with IL-6 expression in osteosarcoma tissues. IL-6 exposure to osteosarcoma cells significantly increased the mRNA and protein expression of SOX18 and the phosphorylation of STAT3 in a dose-dependent manner. Our study suggested that IL-6/STAT3 regulated SOX18 expression in osteosarcoma, although the detailed mechanisms remain to be elucidated.

Furthermore, the involvement of SOX18 in IL-6-affected osteosarcoma cell proliferation and apoptosis was also studied. CCK-8 assays showed that IL-6 treatment significantly promoted osteosarcoma cell proliferation. Knockdown of SOX18 significantly suppressed the effects of IL-6 on cell proliferation. Although IL-6 had little effect on cell 
apoptosis, it could significantly reduce SOX18 knockdowninduced cell apoptosis. These findings revealed that IL-6 is a possible upstream regulator for SOX18. PCNA is a well-accepted marker for cell proliferation. ${ }^{22} \mathrm{Bcl}-2$ family proteins can either stimulate cell survival (eg, Bcl-2) or induce cell apoptosis (eg, Bax). ${ }^{23}$ Cleaved-Caspase 3 is a marker for cell apoptosis. ${ }^{24}$ Here, the change trends of these four proteins were consistent with the results of CCK-8 and cell apoptosis assays.

\section{Conclusion}

IL-6 treatment significantly enhanced SOX18 expression in osteosarcoma. IL-6 rescued the inhibitory and induction effects of SOX18 knockdown on osteosarcoma cell proliferation and apoptosis, respectively. Our study may provide insights to advance our understandings on the occurrence and development of osteosarcoma.

\section{Disclosure}

The authors report no conflicts of interest in this work.

\section{References}

1. Ottaviani G, Jaffe N. The epidemiology of osteosarcoma. Cancer Treat Res. 2009;152:3-13.

2. Ferguson WS, Goorin AM. Current treatment of osteosarcoma. Cancer Invest. 2001;19(3):292-315.

3. Hosking BM, Muscat GE, Koopman PA, Dowhan DH, Dunn TL. Trans-activation and DNA-binding properties of the transcription factor, Sox-18. Nucleic Acids Res. 1995;23(14):2626-2628.

4. Hosking BM, Wyeth JR, Pennisi DJ, Wang SC, Koopman P, Muscat GE. Cloning and functional analysis of the Sry-related HMG box gene, Sox 18 . Gene. 2001;262(1-2):239-247.

5. Dong C, Wilhelm D, Koopman P. Sox genes and cancer. Cytogenet Genome Res. 2004;105(2-4):442-447.

6. Eom BW, Jo MJ, Kook MC, et al. The lymphangiogenic factor SOX 18: a key indicator to stage gastric tumor progression. Int J Cancer. 2012; 131(1):41-48.

7. Wang Y, Guo H, Zhang D, et al. Overexpression of SOX18 correlates with accelerated cell growth and poor prognosis in human pancreatic ductal adenocarcinoma. Biochem Biophys Res Commun. 2016;479(3): 510-516.

8. Pula B, Olbromski M, Wojnar A, et al. Impact of SOX18 expression in cancer cells and vessels on the outcome of invasive ductal breast carcinoma. Cell Oncol (Dordr). 2013;36(6):469-483.
9. Pula B, Kobierzycki C, Solinski D, et al. SOX18 expression predicts response to platinum-based chemotherapy in ovarian cancer. Anticancer Res. 2014;34(8):4029-4037.

10. Jethon A, Pula B, Olbromski M, et al. Prognostic significance of SOX18 expression in non-small cell lung cancer. Int J Oncol. 2015;46(1): $123-132$.

11. Wu Z, Liu J, Wang J, Zhang F. SOX18 knockdown suppresses the proliferation and metastasis, and induces the apoptosis of osteosarcoma cells. Mol Med Rep. 2016;13(1):497-504.

12. Hodge DR, Hurt EM, Farrar WL. The role of IL-6 and STAT3 in inflammation and cancer. Eur J Cancer. 2005;41(16):2502-2512.

13. Williams JG. STAT signalling in cell proliferation and in development. Curr Opin Genet Dev. 2000;10(5):503-507.

14. Battle TE, Frank DA. The role of STATs in apoptosis. Curr Mol Med. 2002;2(4):381-392.

15. Bromberg JF, Wrzeszczynska MH, Devgan G, et al. Stat3 as an oncogene. Cell. 1999;98(3):295-303.

16. Ryu K, Choy E, Yang C, et al. Activation of signal transducer and activator of transcription 3 (Stat3) pathway in osteosarcoma cells and overexpression of phosphorylated-Stat 3 correlates with poor prognosis. J Orthop Res. 2010;28(7):971-978.

17. Foshay KM, Gallicano GI. Regulation of Sox 2 by STAT3 initiates commitment to the neural precursor cell fate. Stem Cells Dev. 2008; 17(2):269-278.

18. Yang J, Liao D, Chen C, et al. Tumor-associated macrophages regulate murine breast cancer stem cells through a novel paracrine EGFR/Stat3/ Sox-2 signaling pathway. Stem Cells. 2013;31(2):248-258.

19. Okamoto M, Lee C, Oyasu R. Interleukin-6 as a paracrine and autocrine growth factor in human prostatic carcinoma cells in vitro. Cancer Res. 1997;57(1):141-146.

20. Okamoto M, Hattori K, Oyasu R. Interleukin-6 functions as an autocrine growth factor in human bladder carcinoma cell lines in vitro. Int $J$ Cancer. 1997;72(1):149-154.

21. Miki S, Iwano M, Miki Y, et al. Interleukin-6 (IL-6) functions as an in vitro autocrine growth factor in renal cell carcinomas. FEBS Lett. 1989;250(2):607-610.

22. Kubben FJ, Peeters-Haesevoets A, Engels LG, et al. Proliferating cell nuclear antigen (PCNA): a new marker to study human colonic cell proliferation. Gut. 1994;35(4):530-535.

23. Reed JC. Regulation of apoptosis by bcl-2 family proteins and its role in cancer and chemoresistance. Curr Opin Oncol. 1995;7(6):541-546.

24. Seki K, Yoshikawa H, Shiiki K, Hamada Y, Akamatsu N, Tasaka K. Cisplatin (CDDP) specifically induces apoptosis via sequential activation of caspase-8, -3 and -6 in osteosarcoma. Cancer Chemother Pharmacol. 2000;45(3):199-206.

25. Kuwahara M, Yamashita M, Shinoda K, et al. The transcription factor Sox4 is a downstream target of signaling by the cytokine TGF-[beta] and suppresses TH2 differentiation. Nat Immunol. 2012;13(8):778-786.

26. Lin Y-M, Chang Z-L, Liao Y-Y, Chou M-C, Tang C-H. IL-6 promotes ICAM-1 expression and cell motility in human osteosarcoma. Cancer Lett. 2013;328(1):135-143.
OncoTargets and Therapy

\section{Publish your work in this journal}

OncoTargets and Therapy is an international, peer-reviewed, open access journal focusing on the pathological basis of all cancers, potential targets for therapy and treatment protocols employed to improve the management of cancer patients. The journal also focuses on the impact of management programs and new therapeutic agents and protocols on

\section{Dovepress}

patient perspectives such as quality of life, adherence and satisfaction. The manuscript management system is completely online and includes a very quick and fair peer-review system, which is all easy to use. Visit http://www.dovepress.com/testimonials.php to read real quotes from published authors. 